\section{Índice de Saúde Aplicado ao Município de Araraquara, SP: um instrumento para o acompanhamento da Atenção Básica}

\section{Health Index applied to the city of Araraquara, SP: an instrument to accompaniment the Primary Care}

\author{
Augustus Tadeu Relo de Mattos' \\ Maria do Carmo G.G. Caccia-Bava' \\ Débora Cristina Modesto Barbosa" \\ ' Departamento de Medicina Social da Faculdade de Medicina de Ribeirão Preto \\ da Universidade de São Paulo. \\ "Universidade de Franca - UNIFRAN
}

Responsabilidade individual dos autores:

Augustus Tadeu Relo de Mattos foi responsável pela concepção do artigo e por seu desenvolvimento a partir de revisão crítica de literatura apresentada, articulação teórico-metodológica apresentada, revisão crítica de conteúdo e aprovação final do artigo.

Maria do Carmo G. G. Caccia-Bava foi responsável pela ampliação da revisão bibliográfica acerca da temática e pela revisão crítica do conteúdo do artigo, auxiliando na articulação teórico-metodológica.

Débora Cristina Modesto Barbosa foi responsável pela revisão da literatura e pela organização das referências bibliográficas.

O texto é inédito, não houve financiamento, e é resultado de dissertação de mestrado, não haven do conflitos de interesse. Foi aprovado por CEP sob o parecer 183/ CEP-CSE-FMRP-USP.

Correspondência: Augustus Tadeu Relo de Mattos. Departamento de Medicina Social, Faculdade de Medicina de Ribeirão Preto, USP. Rua Paschoal Bardaro, ${ }^{\circ}$ 2115, ap. 22 - Jardim Botânico, Ribeirão Preto, SP CEP 14021-655. E-mail: augustus@fmrp.usp.br

\section{Resumo}

O aprimoramento do processo de avaliação da Atenção Básica, compreendida como organizadora de todo o sistema de saúde e não apenas como um nível assistencial, vem sendo almejado pelos gestores do Sistema Único de Saúde. O presente trabalho buscou identificar aspectos da assistência à saúde no município de Araraquara/SP, valorizando as características particulares presentes na área de adscrição de cada unidade de saúde. Adotando um indicador composto aplicado a cada unidade de saúde, o estudo visou contemplar a emergência de fenômenos favorecendo uma visão mais abrangente de cada unidade e distrito sanitário em relação à Mortalidade Infantil, Incidência de Tuberculose, Mortalidade por Causas Externas e Mortalidade Precoce por Doenças Crônicas. Valeu-se da metodologia desenvolvida no Índice de Saúde de Drumond Jr, obtido pela média dos valores dos componentes de cada Distrito de Saúde para os indicadores acima, agregando, ainda, a Taxa de Alfabetização e Rendimento Médio Mensal do Responsável pelo Domicílio. As áreas de abrangência das Unidades de Saúde foram classificadas segundo os valores obtidos na relação entre estes componentes. Os resultados apontaram diferenciais intraurbanos para a maioria dos componentes e revelou a presença dos piores valores nas Unidades de Saúde da periferia. O conhecimento dessa realidade pela gestão municipal é de fundamental importância para avançar no aprimoramento do processo de avaliação da Atenção Básica no município.

Palavras-chave: Avaliação em saúde. Atenção primária à saúde. Indicadores básicos de saúde. Desigualdades em saúde. 


\section{Abstract}

The improvement of the process of evaluation and monitoring of the Primary Care Attention has been yearned by the managers of the different spheres in the context of the Unified System Health - UHS. Thus, in the order to identify the conditions of the health assistance in the city of Araraquara/SP, based on the particular features presented in the inscribe areas of each unit of health. It was adopted by means of a composed indicator aiming to favor a broader view which contemplates the emergency of phenomena from a more including vision. It was used the methodology developed in the Index of Health of Drumond Jr, whose final value of the Index is gotten by the average of the values of the components of each health district. To the components originally adopted - Coefficient of Infant Mortality, Coefficient of Tuberculosis Incidence, Coefficient of Mortality by External Causes and Coefficient of Precocious Mortality by Chronic Diseases - was still added in this study, the Tax of Alphabetization and the Monthly Nominal Average Income of Responsible People for the Domiciles. The 24 Units of Health had been classified according to values obtained in the relation among its components. The results point intra-urban differentials in relation to the majority of the components and lower values for the Health Units located at the periphery of the city. Knowing this fact municipal administration is of fundamental importance to progress in improving the process of evaluation of primary care in the county.

Keywords: Health evaluation. Primary health care. Health status indicators. Health inequalities.

\section{Introdução}

O aprimoramento do processo de avaliação e monitoramento das ações de atenção básica, aqui compreendida como organizadora de todo o sistema de saúde e não apenas um nível assistencial, vem sendo almejado pelos gestores das diversas esferas do SUS. Como um instrumento da gestão, a avaliação permite estabelecer bases para o planejamento de novas ações, corrigindo rumos e aperfeiçoando os processos de forma a se obter os objetivos esperados ${ }^{1}$.

Para Mendes ${ }^{2}$, o modelo de avaliação tradicional só poderá ser superado com a incorporação de novas variáveis, como indicadores de qualidade e decisões de fóruns institucionais (como conselhos locais e municipais de saúde), utilizados como potentes recursos de articulação e execução da política municipal de saúde, viabilizando o atendimento às necessidades e racionalizando gastos a partir do efetivo controle social.

A Atenção Básica adotou como uma importante forma de avaliação, o Pacto dos Indicadores da Atenção Básica, iniciado em 1999, hoje incorporando as dimensões do Pacto pela Saúde, que consistem em um dos principais instrumentos de acompanhamento e avaliação de abrangência nacional, estabelecido a partir da negociação de metas, que representam a intenção dos gestores de melhorar a qualidade da atenção à saúde ${ }^{3}$.

Entretanto, as iniquidades presentes na nossa sociedade imprimem diferenças - evitáveis, sistemáticas e injustas - nas condições de vida e saúde das pessoas presentes nos diferentes bairros das várias cidades e regiões brasileiras, que podem ser constatadas na distribuição dos agravos à saúde. Dessa forma, pode-se concluir que as populações não se distribuem por acaso nas unidades territoriais de uma cidade, mas tendem a formar conglomerados humanos que compartilham características relativamente similares de natureza social e econômica. Essas diferenças criam espaços segregados que mudam, cotidianamente, 
a vida nesses locais e, em consequência, transformam a vida da cidade como um todo $^{4}$.

A articulação de vários indicadores sociais na construção de um índice de avaliação composto pode permitir uma análise particularizada da assistência na área de abrangência das Unidades de Saúde, onde a desagregação dos dados a partir da realidade local permite a identificação das necessidades de intervenções diferenciadas entre os diversos territórios, justificando investimentos priorizados nas áreas onde os problemas tenham maior relevância sócio-sanitária.

A desagregação geográfica mínima deve representar um compromisso entre obter uma área reduzida o suficiente para ser homogênea e extensa o bastante para fornecer número adequado de eventos para análise ${ }^{4}$ e que discriminem diferentes necessidades.

Dessa forma, justifica-se que a análise de dados epidemiológicos seja desagregada entre territórios de uma mesma cidade para que essas diferenças possam ser consideradas na formulação de políticas compatíveis com a diversidade intra-urbana ${ }^{5}$, considerando-se que a exclusão social é a impossibilidade de partilhar os benefícios produzidos pela sociedade, levando à vivência da privação, da recusa, do abandono e da expulsão.

Não se trata de um processo individual, embora atinja pessoas, mas de uma lógica que está presente nas várias formas de relações econômicas, sociais, culturais e políticas. Essa situação de privação coletiva é o que se entende por exclusão social ${ }^{6}$, que, segundo o relatório mundial da OMS, deriva de uma estrutura social que gera desigualdades que atingem também as desigualdades relacionadas aos sistemas de saúde.

Os serviços de saúde, em sua maioria, desde hospitais até as unidades de cuidados primários, apresentam formas muito distintas na prestação da assistência particularmente no que se refere à qualidade, produzindo desigualdade sobretudo para aqueles que mais necessitam ${ }^{7}$.

Na procura por inovações metodológicas que possam contemplar a variedade de condições que estão relacionadas às iniquidades em saúde, vislumbra-se a utilização de um índice composto que contemple essa pluralidade, evidenciando as desigualdades num dado território, e auxiliar no planejamento, monitoramento e avaliação das ações em saúde, direcionando as intervenções para diminuir tais iniquidades ${ }^{8}$.

Um indicador composto é uma medida que associa diferentes variáveis numa referência sintética para analisar as características de grupos populacionais em determinadas áreas geográficas. Por ser composto, permite a pluralidade de perspectivas na sua elaboração ${ }^{9}$.

Para Mendes², os indicadores compostos são instrumentos que possibilitam demonstrar fenômenos em áreas geográficas específicas, classificando-as em variáveis que reflitam as circunstâncias materiais ou sociais do fenômeno que se quer examinar. A distribuição da população no espaço urbano segue os padrões de desigualdade: de um lado, a periferia da cidade, sem infraestrutura urbana, ocupada por grupos menos privilegiados, de baixa renda e em piores condições de saúde; do outro lado existem áreas com acesso total às facilidades urbanas que são ocupadas por grupos de alta renda e com melhores padrões de saúde ${ }^{4}$. Um exemplo de indicador composto é o Índice Paulista de Vulnerabilidade Social (IPVS), que permite uma visão mais precisa das condições de vida, identificando espacialmente os segmentos populacionais mais sujeitos à pobreza e outros fatores de vulnerabilidade. Para tanto utiliza como parâmetros as dimensões socioeconômica e do ciclo de vida familiar ${ }^{10}$.

Um estudo que considera a desigualdade e exclusão social, bem como a qualidade de vida, a partir de dados epidemiológicos não se constitui apenas em um instrumento de diagnóstico, mas de intervenção, justificando o desenvolvimento deste trabalho que tem como objetivo analisar as condições de saúde do município de Araraquara/ SP, no ano de 2004, a partir da aplicação do Índice de Saúde ${ }^{11}$ modificado. 


\section{Metodologia}

Trata-se de um estudo descritivo, realizado no município de Araraquara/SP, concebido a partir da aplicação de uma nova metodologia, baseada no Índice de Saúde de Drumond Jr. ${ }^{11}$, cujos componentes são: Coeficiente de Mortalidade Infantil (CMI), Coeficiente de Incidência de Tuberculose (CIT), Coeficiente de Mortalidade por Causas Externas (CMCE) e Coeficiente de Mortalidade Precoce por Doenças Crônicas não Transmissíveis (CMDC) na população entre 20 e 59 anos. Segue-se a metodologia utilizada na construção do Índice de Desenvolvimento Humano (IDH), que é um indicador composto, geral e sintético.

Foram incorporados ao Índice de Saúde de Drumond Jr. dois novos componentes: Taxa de Alfabetização de Pessoas Responsáveis Domicílios (TARD) e Rendimento Médio Nominal de Pessoas Responsáveis pelos Domicílios (RMRD), levando a um novo índice denominado Índice de Saúde Modificado (ISM). A opção pela inclusão desses dois indicadores veio no sentido de particularizar ainda mais o conhecimento sobre as características envolvidas no processo saúde-doença, presentes nos diferentes territórios.

Para o CMI a base populacional clássica é de mil nascidos vivos e, para o CIT, o CMCE e o CMDC utilizou-se base populacional de 100 mil.

No cômputo do ISM foram calculadas a distância e a discrepância. A distância é a medida entre o valor obtido pela Unidade de Saúde e o pior valor encontrado no município, enquanto a discrepância é a diferença entre o maior e o pior valor obtidos no município. De maneira conceitual essa relação aponta como a situação real de uma determinada área de abrangência correspondente a uma Unidade de Saúde em análise se encontra em relação ao ideal desejado para a condição de saúde do município ${ }^{12}$.

Para Samohyl ${ }^{13}$, a distância pode ser entendida como a amplitude geral da série de dados (a amplitude é uma medida de dispersão calculada como a diferença entre o maior e o menor valor de um conjunto de dados) e a discrepância como uma medida que procura estabelecer um grau de aproximação com as condições concretas de vida da população nos diferentes territórios de uma cidade ou região, traduzindo quantitativamente e demonstrando o tamanho das desigualdades existentes ${ }^{14}$.

O valor de cada componente foi obtido pela razão distância/discrepância. Sendo assim, foi possível construir um índice sintético cujo resultado tem o intuito de identificar as desigualdades no território estudado ${ }^{14}$.

Esta formulação foi aplicada aos seis componentes acima elencados para cada uma das 24 Unidades de Saúde, sendo que o valor do índice deve estar entre zero e um. Sendo assim, a Unidade de Saúde com pior situação obteve valor zero e a com melhor situação recebeu o valor um para cada um dos seis componentes do ISM. As demais Unidades de Saúde apresentaram valores intermediários. Por fim, o valor atribuído ao ISM foi computado como a média aritmética dos valores obtidos para cada um dos seis componentes.

A agregação desses seis componentes reflete aspectos prioritários da política de saúde, incluindo alguns aspectos econômicos e sociais que, no seu conjunto, pretendem sintetizar uma realidade que abrange condições de vida e saúde. Deve-se ressaltar que essa metodologia é adequada para comparar diferenciais intraurbanos no município, mas não para a comparação entre cidades e regiões. Ela nos permite observar, ao longo dos anos, o desempenho ou a mobilidade das áreas estudadas a partir de intervenções ou mudanças realizadas ${ }^{11}$. Assim como na proposta de Drumond Jr., neste estudo não se atribuiu pesos diferenciados aos indicadores, já que todos contemplam aspectos relevantes para a saúde.

A área de responsabilidade de cada unidade de saúde é definida pela Secretaria Municipal de Saúde de Araraquara (SMS) a partir da confluência de vários critérios: contingente populacional a ser assistido pela unidade, densidade tecnológica e capacidade instalada da unidade e tradição 
cultural da população em ser assistida em determinado local. As USFs seguem o parâmetro do Ministério da Saúde de uma equipe para cada mil famílias ou cerca de 3.500 pessoas. O território de cada unidade tem seus limites geográficos definidos, sendo que os setores censitários são utilizados como base para a definição do contingente populacional. As informações epidemiológicas geradas pelos sistemas de informação oficiais da SMS de Araraquara permitiram o seu agrupamento segundo a área de abrangência de cada unidade de saúde, de onde foram extraídos os dados. O local do estudo, Araraquara/SP, é sede da direção regional de saúde - DRS III - composta por 25 municípios para os quais é referência. $\mathrm{O}$ município encontra-se estruturado em três Unidades Distritais de Saúde. Está localizado no centro do Estado de São Paulo, tendo uma população estimada, no ano de 2004, de 191.896 habitantes. Apresentou taxa de alfabetização de $94,8 \%$ no ano de 2000 . Há que se ressaltar os registros existentes na literatura ${ }^{15,16}$, apontando as falhas em todas as etapas do processo de geração de informações, desde a alimentação do sistema até sua utilização. $\mathrm{O}$ grande número de óbitos por causas mal definidas pode exemplificar essas fragilidades no sistema de informação sobre os indicadores relacionados à mortalidade. Essas falhas podem gerar inconsistências nos resultados obtidos quando não identificadas. Aspectos Éticos: após aprovação por Comitê de Ética em Pesquisa e da Secretaria Municipal de Saúde de Araraquara, SP, foram coletados os dados referentes ao período de Janeiro a Dezembro de 2004, a partir da Divisão de Saúde Pública da SMS. Foram também utilizados dados do censo de 2000 do IBGE.

O Quadro 1, abaixo, apresenta uma síntese das áreas de abrangência, as respectivas populações, os valores absolutos para cada componente e a sua classificação no ISM.

\section{Resultados}

Ressaltando que a relação distância/ discrepância busca apontar como o estado real de uma unidade de saúde em análise se encontra em relação ao ideal desejado para a condição de saúde do município, são apresentados, a seguir, os resultados obtidos para cada componente analisado.

- O Coeficiente de Mortalidade Infantil CMI - foi de 9,78 por mil nascidos vivos, apresentando uma diminuição quando comparado aos valores encontrados em estudo de Mattos e Caccia-Bava ${ }^{17}$, que apontou a ocorrência de 11,46 óbitos por 1.000 nascidos vivos no ano de 2002 . Dentre as unidades de saúde do município constatou-se uma variação de 0 e 29,85 óbitos por mil nascidos vivos. Para o cálculo do Coeficiente de Mortalidade Infantil em 2004 foram considerados 2.277 nascimentos.

- O Coeficiente de Mortalidade Precoce por Doenças Crônicas - CMDC - foi de 100,70 óbitos por 100 mil habitantes. Dos 265 óbitos por Hipertensão Arterial Sistêmica (HAS) e Diabetes na população acima de 20 anos, 100 ocorreram na população entre 20 e 59 anos, sendo 91 relacionados à HAS e nove ao Diabetes. Dentre esses 100 óbitos, quatro ocorreram na população da zona rural. Os 165 óbitos restantes ocorreram na população com mais de 59 anos. Nesse estudo foram excluídos os óbitos relacionados a Hipertensão e ao Diabetes Mellitus ocorridos na população rural, num total de quatro óbitos, em uma população de 6.984 habitantes. Estes óbitos foram excluídos por não estarem formalmente adscritos a nenhuma das 24 Unidades de Saúde do município, dificultando a vinculação do evento a uma unidade de saúde específica. Portanto o número considerado para o cálculo desse indicador foi de 96 óbitos, que corresponde ao total de óbitos por Hipertensão Arterial Sistêmica e Diabetes Mellitus.

- OCoeficiente de Mortalidade por Causas Externas - CMCE - foi de 51,59 por 100 mil habitantes, variando entre 0 e 109,7 óbitos por 100 mil habitantes. O número total de óbitos por causas externas foi de 99, sendo dois na zona rural e sete 
Quadro 1 - Síntese das áreas de abrangência, as respectivas populações, os valores absolutos para cada componente e a respectiva classificação no ISM, Araraquara, 2004.

Chart 1 - Summary of catchment areas, their populations, the absolute values for each component and their classification in the ISM, Araraquara, 2004.

\begin{tabular}{|c|c|c|c|c|c|c|c|c|c|}
\hline \multirow{3}{*}{$\begin{array}{l}\text { Áreas de abrangência das } \\
\text { unidades de saúde }\end{array}$} & \multirow{3}{*}{ População } & \multicolumn{7}{|c|}{ Componentes e seus respectivos números absolutos* } & \multirow{3}{*}{ ISM } \\
\hline & & \multirow{2}{*}{ Ol } & \multirow{2}{*}{ CNTB } & \multicolumn{2}{|c|}{ OPDC } & \multirow{2}{*}{ OCE } & \multirow{2}{*}{ NPRDA } & \multirow{2}{*}{ RMRD } & \\
\hline & & & & HAS & DIA & & & & \\
\hline SESA & 29192 & 1 & 3 & 16 & 1 & 17 & 8.927 & 1781,83 & $1^{\circ}$ \\
\hline CMS PAULISTANO & 14645 & 0 & 2 & 4 & 0 & 3 & 3.958 & 1012,69 & $2^{\circ}$ \\
\hline PSF B. ANDRADA & 1810 & 0 & 0 & 0 & 0 & 0 & 384 & 318,00 & $2^{\circ}$ \\
\hline PSF BELA VISTA & 834 & 0 & 0 & 0 & 0 & 0 & 170 & 438,00 & $3^{\circ}$ \\
\hline PSF MARIVAN & 6237 & 0 & 0 & 2 & 0 & 4 & 1.798 & 729,39 & $4^{\circ}$ \\
\hline CMS S. ANGELINA & 17119 & 1 & 6 & 8 & 1 & 5 & 4.825 & 1127,84 & $5^{\circ}$ \\
\hline CMS SANTA LÚCIA & 11657 & 1 & 3 & 2 & 0 & 3 & 3.187 & 1037,98 & $5^{\circ}$ \\
\hline PSF VALE DO SOL & 4091 & 0 & 2 & 4 & 0 & 0 & 1.032 & 627,81 & $6^{\circ}$ \\
\hline CMS SELMI DEI IV & 6623 & 0 & 1 & 2 & 0 & 2 & 1.657 & 578,30 & $6^{\circ}$ \\
\hline CMS CECAP & 5315 & 0 & 0 & 2 & 0 & 5 & 1.343 & 626,65 & 70 \\
\hline PSF HORTENSIAS & 3486 & 0 & 0 & 2 & 0 & 0 & 695 & 302,89 & $8^{\circ}$ \\
\hline CMS JD. AMERICA & 15624 & 2 & 4 & 4 & 0 & 9 & 3.988 & 734,66 & $9^{\circ}$ \\
\hline CMS JD. IGUATEMI & 3532 & 0 & 2 & 2 & 0 & 2 & 930 & 659,98 & $10^{\circ}$ \\
\hline CMS V. MELHADO & 9009 & 3 & 2 & 2 & 1 & 3 & 2.387 & 1038,16 & $10^{\circ}$ \\
\hline CMS LARANJEIRAS & 7068 & 1 & 4 & 2 & 1 & 4 & 1.846 & 875,08 & $11^{\circ}$ \\
\hline CMS VILA XAVIER & 13792 & 2 & 9 & 8 & 2 & 9 & 3.958 & 942,34 & $12^{\circ}$ \\
\hline PSF JD. PINHEIROS & 5228 & 0 & 1 & 2 & 0 & 3 & 792 & 577,54 & $13^{\circ}$ \\
\hline PSF MARIA LUIZA & 1822 & 1 & 0 & 2 & 0 & 1 & 434 & 602,14 & $14^{\circ}$ \\
\hline CMS Y. ÓPICE & 7030 & 3 & 2 & 6 & 1 & 4 & 1.648 & 596,46 & $15^{\circ}$ \\
\hline CMS SELMI DEI I & 7287 & 4 & 3 & 0 & 0 & 8 & 1.715 & 497,99 & $16^{\circ}$ \\
\hline PSF A. DO PAIOL & 2380 & 2 & 1 & 2 & 0 & 0 & 525 & 397,04 & $17^{\circ}$ \\
\hline PSF V. BIAGIONI & 1743 & 1 & 1 & 1 & 0 & 1 & 469 & 503,20 & $18^{\circ}$ \\
\hline PSF IEDA & 3887 & 0 & 3 & 10 & 0 & 3 & 853 & 395,46 & $19^{\circ}$ \\
\hline CMS PQ.S. PAULO & 5509 & 1 & 5 & 6 & 0 & 4 & 1.240 & 494,35 & $20^{\circ}$ \\
\hline TOTAL & 183920 & 23 & 54 & 89 & 7 & 90 & - & - & - \\
\hline
\end{tabular}

*OI - Número de Óbitos em Menores de um ano, CNTB - Número de Casos Novos de Tuberculose, OPDC - Número de Óbitos Precoces doenças crônicas (aqui considerados os óbitos relacionados à Hipertensão Arterial Sistêmica - HAS - e ao Diabetes - DIA - na população de 20 a 59 anos), OCE - Número de Óbitos por Causas Externas, NPRDA -Número de Pessoas Responsáveis pelos Domicílios Alfabetizadas, RMRD - Rendimento Médio de Pessoas Responsáveis pelos Domicílios.

${ }^{*} D C$ - Number of deaths in children younger than one year, CNTB - Number of new cases of tuberculosis, EDCD - Number of early deaths from chronic diseases (deaths associated with Systemic Arterial Hypertension - SAH - and Diabetes - DIA - in the population aged between 20 and 59 years were taken into consideration here), DEC - Number of deaths from external causes, $\mathrm{NLHH}$ - Number of literate heads of household, HHAI-Heads of household average income.

em bairros ignorados, face a impossibilidade do sistema de informações da Secretaria Municipal de Saúde vincular estes eventos a uma unidade de saúde.

- O Coeficiente de Incidência de Tuberculose - CIT - foi de 30,22 casos de tuberculose por 100 mil habitantes. Entre as Unidades de Saúde, variou de zero a 90,7 casos de tuberculose por 100 mil habitantes. Para o Estado de São Paulo no ano de 2004, a Incidência de Tuberculose foi de 45,9 casos por 100 mil habitantes segundo o Centro de Vigilância Epidemiológica Alexandre Vranjac ${ }^{18}$.

- O Rendimento Médio Nominal de
Pessoas Responsáveis pelos Domicílios - RMRD - apontou valores variando entre $\mathrm{R} \$ 302,89$ e $\mathrm{R} \$ 1.781,83$ na área de abrangência das 24 unidades de saúde, conforme dados do Censo Demográfico do ano 2000.

- A Taxa de Alfabetização de Pessoas Responsáveis Domicílios - TARD - calculada pelo percentual de pessoas com mais de 15 anos capazes de ler e escrever um bilhete simples variou entre $68,2 \mathrm{e}$ $97,8 \%$ no ano de 2000 , conforme dados do Censo Demográfico do ano 2000.

Após a aplicação da relação distância/ 
discrepância para cada componente do estudo para em seguida calcular a média aritmética entre estes componentes, encontraram-se os valores do ISM de cada Unidade de Saúde com os resultados variando entre 0,38 (pior desempenho) e 0,81 (melhor desempenho).

\section{Discussão}

Áreas com pequenas populações (e, por conseguinte, poucos óbitos) são suscetíveis a grande variabilidade nas taxas de mortalidade ou em outros indicadores, decorrente de flutuações aleatórias. Estas flutuações podem interferir nos resultados quando se analisa curtos períodos de tempo. Assim, determinada área pode ter sua classificação alterada drasticamente, de um ano para outro, razão pela qual se buscou apresentar os valores absolutos de cada componente, além da sua classificação individual, antes de apresentar a síntese dos indicadores no ISM.

Após aplicação da metodologia do ISM considerando os seis componentes adotados para análise, obteve-se a classificação final das Unidades de Saúde, conforme apontado pelo Quadro 2 a seguir e sua

Quadro 2 - Resultados obtidos a partir da relação distância/discrepância aplicada para cada componente e classificação das Unidades de Saúde e respectivas áreas de abrangência no Índice de Saúde de Drumond Jr. Modificado (ISM), Araraquara, 2004.

Chart 2 - Results obtained from the distance/discrepancy ratio for each component and classification of the Health Units and their coverage areas in the Drumond Jr. Modified Health Index, Araraquara, 2004.

\begin{tabular}{|lccccccccc|}
\hline \multirow{2}{*}{$\begin{array}{l}\text { Áreas de abrangência das } \\
\text { unidades de saúde }\end{array}$} & \multicolumn{7}{c}{ Valores obtidos para os componentes do índice de saúde } & Índice de saúde & Classif. \\
& CMI & CIT & CMDC & CMCE & RMRD & TARD & modificado & Final \\
\cline { 2 - 6 } SESA & 0,81 & 0,88 & 0,73 & 0,46 & 1 & 0,98 & 0,81 & $1^{\circ}$ \\
CMS PAULISTANO & 1 & 0,85 & 0,75 & 0,81 & 0,48 & 0,93 & 0,80 & $2^{\circ}$ \\
PSF B. ANDRADA & 1 & 1 & 1 & 1 & 0,01 & 0,76 & 0,80 & $2^{\circ}$ \\
PSF BELA VISTA & 1 & 1 & 1 & 1 & 0,09 & 0,47 & 0,76 & $3^{\circ}$ \\
PSF MARIVAN & 1 & 1 & 0,71 & 0,41 & 0,29 & 0,96 & 0,73 & $4^{\circ}$ \\
CMS S. ANGELINA & 0,83 & 0,61 & 0,64 & 0,73 & 0,56 & 0,96 & 0,72 & $5^{\circ}$ \\
CMS SANTA LÚCIA & 0,68 & 0,71 & 0,8 & 0,76 & 0,50 & 0,85 & 0,72 & $5^{\circ}$ \\
PSF VALE DO SOL & 1 & 0,47 & 0,43 & 1 & 0,22 & 0,92 & 0,67 & $6^{\circ}$ \\
CMS SELMI DEI IV & 1 & 0,83 & 0,33 & 0,72 & 0,19 & 0,96 & 0,67 & $6^{\circ}$ \\
CMS CECAP & 1 & 1 & 0,67 & 0,14 & 0,22 & 0,93 & 0,66 & $7^{\circ}$ \\
PSF HORTENSIAS & 1 & 1 & 0,33 & 1 & 0 & 0,51 & 0,64 & $8^{\circ}$ \\
CMS JD. AMERICA & 0,68 & 0,71 & 0,73 & 0,47 & 0,29 & 0,83 & 0,62 & $9^{\circ}$ \\
CMS JD. IGUATEMI & 1 & 0,38 & 0,50 & 0,48 & 0,24 & 1 & 0,60 & $10^{\circ}$ \\
CMS V. MELHADO & 0,08 & 0,75 & 0,63 & 0,69 & 0,50 & 0,92 & 0,60 & $10^{\circ}$ \\
CMS LARANJEIRAS & 0,7 & 0,37 & 0,67 & 0,48 & 0,39 & 0,89 & 0,58 & $11^{\circ}$ \\
CMS VILA XAVIER & 0,63 & 0,28 & 0,71 & 0,4 & 0,43 & 0,94 & 0,57 & $12^{\circ}$ \\
PSF JD. PINHEIROS & 1 & 0,78 & 0,6 & 0,47 & 0,19 & 0 & 0,51 & $13^{\circ}$ \\
PSF MARIA LUIZA & 0 & 1 & 0,33 & 0,50 & 0,2 & 0,76 & 0,47 & $14^{\circ}$ \\
CMS Y. ÓPICE & 0,33 & 0,69 & 0,22 & 0,48 & 0,2 & 0,83 & 0,46 & $15^{\circ}$ \\
CMS SELMI DEII & 0,1 & 0,54 & 1 & 0 & 0,13 & 0,81 & 0,43 & $16^{\circ}$ \\
PSF A. DO PAIOL & 0,21 & 0,53 & 0 & 1 & 0,06 & 0,64 & 0,41 & $17^{\circ}$ \\
PSF V. BIAGIONI & 0,58 & 0,36 & 0 & 0,47 & 0,14 & 0,87 & 0,40 & $18^{\circ}$ \\
PSF IEDA & 1 & 0,14 & 0,17 & 0,29 & 0,06 & 0,68 & 0,39 & $19^{\circ}$ \\
CMS PQ.S. PAULO & 0,73 & 0 & 0,4 & 0,33 & 0,13 & 0,68 & 0,38 & $20^{\circ}$ \\
\hline
\end{tabular}

** CMI Coeficiente de Mortalidade Infantil; CIT Coeficiente de Incidência de Tuberculose; CMDC - Coeficiente de Mortalidade Precoce por Doenças Crônicas não Transmissíveis, CMCE - Coeficiente de Mortalidade por Causas Externa;TARD - Taxa de Alfabetização de Pessoas Responsáveis Domicílios; RMRD - Rendimento Médio Nominal de Pessoas Responsáveis pelos Domicílios.

** CMC - Child Mortality Coefficient; TIC - Tuberculosis Incidence Coefficient; MCCD - Early Mortality Coefficient due to Chronic Non-Communicable Diseases, ECMC

- External Cause Mortality Coefficient; HHLR - Head of Household Literacy Rate; and HHAl - Head of Household Nominal Average Income. 


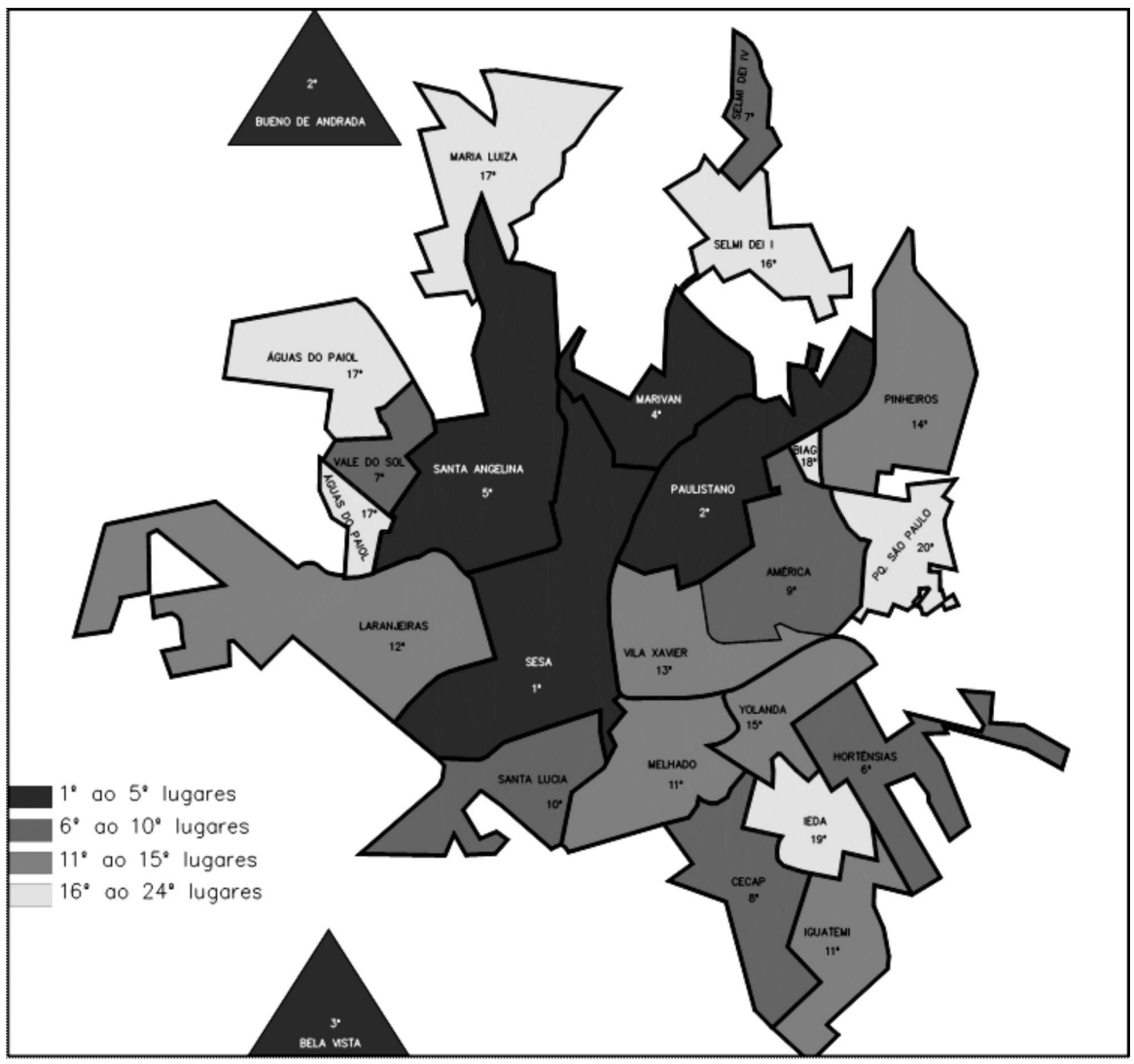

Figura 1 - Representação cartográfica da Síntese dos Componentes por ordem de Classificação das Unidades de Saúde no Índice de Saúde de Drumond Jr. Modificado, segundo área de abrangência, Araraquara, 2004.

Figure 1 - Mapping of the Synthesis of Components in order of Rating Units in Health Health Index Modified for Drummond, Jr., second coverage area, Araraquara, 2004.

representação cartográfica na Figura 1.

A partir dos resultados obtidos podem ser trazidos à nossa reflexão, inicialmente, os seguintes aspectos: dentre as 24 Unidades de Saúde, 11 não apresentaram óbitos infantis e, portanto, necessitariam de uma análise histórica desse indicador. Em estudo semelhante realizado por Drumond Jr. ${ }^{19}$ no município de São Paulo, esse componente variou entre 7,2 e 55,2 óbitos por mil nascidos vivos. Para esse componente verificaram-se importantes diferenciais intraurbanos segundo as áreas de abrangência das Unidades de Saúde. Das cinco Unidades com piores resultados, ou seja, Coeficiente de Mortalidade Infantil acima de 20 óbitos infantis por mil nascidos vivos, quatro unidades localizam-se em regiões periféricas da área urbana (PSF Águas do Paiol, CMS Selmi Dei I, PSF Maria Luiza, CMS Yolanda Ópice).
Também se evidenciou que entre as 11 Unidades de Saúde que não apresentaram óbitos infantis, sete foram Unidades Saúde da Família. Para uma avaliação ainda mais aprofundada desse coeficiente, é necessário considerar que a população das respectivas áreas é pequena, justificando a importância de se realizar comparações ao longo dos anos a partir dessa metodologia.

$\mathrm{Cruz}^{20}$, ao analisar este indicador num estudo que envolveu sete municípios paulistas, verificou que a implantação do PSF foi um dos fatores que contribuiu para a queda das taxas de mortalidade infantil nesses municípios. As áreas de maior vulnerabilidade social encontradas por meio do IPVS para o município de Araraquara - SEADE ${ }^{21}$ coincidem com as áreas de abrangência com piores resultados para o componente mortalidade Infantil, apontando para a 


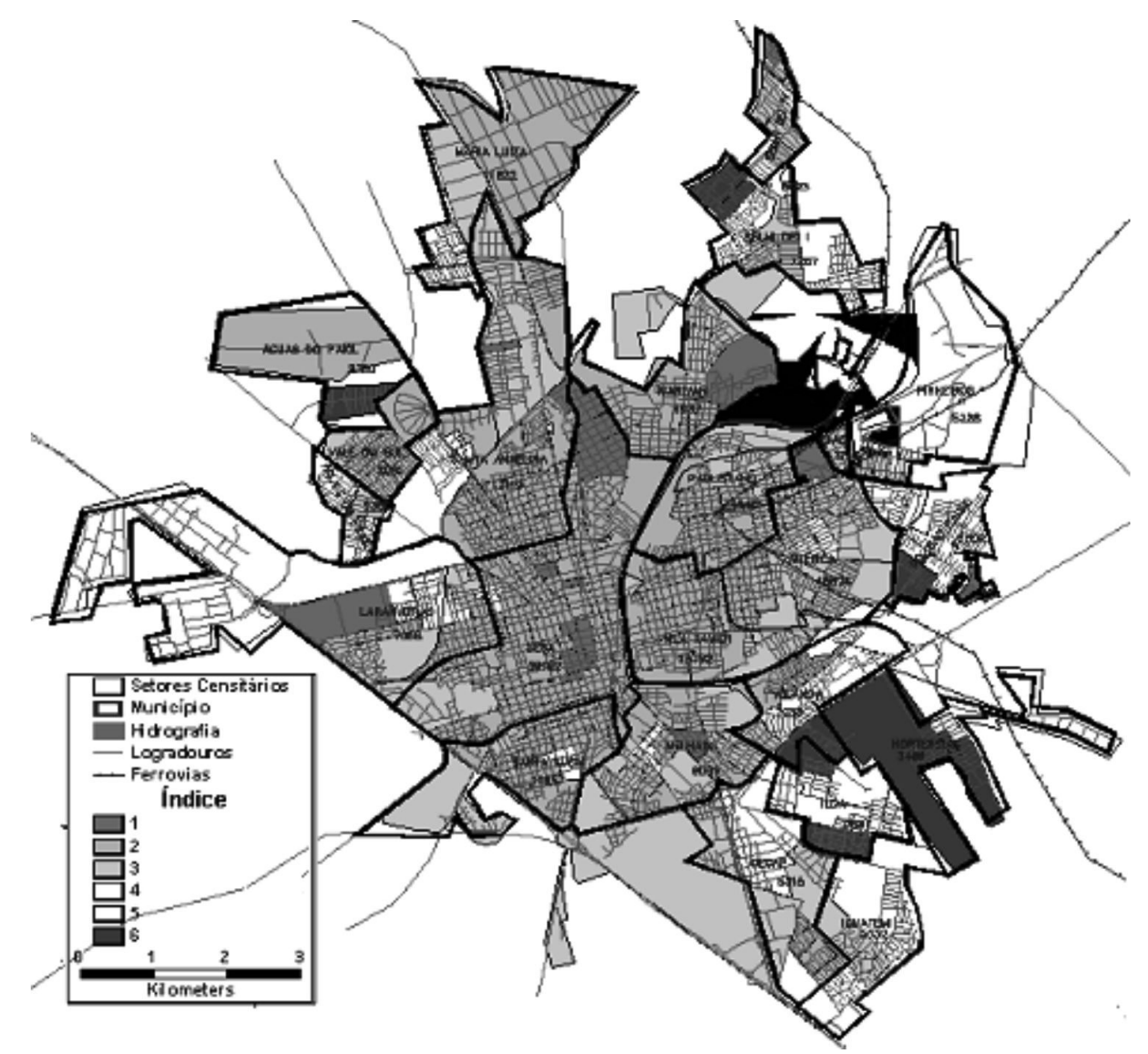

*Sendo atribuídas a cada índice as seguintes classificações: 1 - Nenhuma Vulnerabilidade; 2 - Vulnerabilidade Muito Baixa; 3 - Vulnerabilidade Baixa; 4 - Vulnerabilidade Média; 5 - Vulnerabilidade Alta; 6 Vulnerabilidade Muito Alta.

* The following categories were attributed to each index: 1 - no vulnerability; 2 - very low vulnerability; 3 - low vulnerability; 4 average vulnerability; 5 - high vulnerability; and 6 - very high vulnerability.

Figura 2 - Representação cartográfica do Índice Paulista de Vulnerabilidade Social para a área urbana do município de Araraquara no ano de 2000, modificado com a sobreposição das áreas de abrangência das Unidades de Saúde, no ano de 2004.

Figure 2 - Cartographic representation of the State Social Vulnerability Index for the urban area of Araraquara in 2000, modified with overlapping coverage areas of health units in the year 2004.

distribuição periférica deste evento.

Embora o IPVS adote componentes distintos daqueles empregados pelo ISM, ambos permitem explicitar dimensões relativas ao setor saúde em sua relação com aspectos sociais mais amplos. Desta forma, não se trata de propor a adoção/exclusão de uma referência pela outra, mas da associação de ambas para retratar de forma mais rica uma dada realidade social que é complexa e multifacetária.

A variação do Coeficiente de Incidência de Tuberculose entre as Unidades de Saúde foi de 0 e 90,7 casos de tuberculose por 100 mil habitantes. No estudo realizado por
Drumond Jr. ${ }^{19}$ no município de São Paulo usando a mesma metodologia para as Distritais de Saúde da Capital, esse componente variou entre 32,5 e 113,5 casos de tuberculose por 100 mil habitantes. Entre as Unidades de Saúde que apresentaram os seis piores resultados, ou seja, Coeficiente de Incidência de Tuberculose acima de 50 por 100 mil habitantes estão: CMS Jardim Iguatemi, CMS Laranjeiras, PSF Biagioni, CMS Vila Xavier, PSF Ieda, CMS Parque São Paulo. Quatro dessas unidades localizam-se em regiões periféricas do município e encontram correspondência com as áreas de maior vulnerabilidade social do IPVS. Entre 
as seis unidades que não apresentaram casos de tuberculose, cinco foram Unidades da Estratégia Saúde da Família.

O Coeficiente de Mortalidade Precoce por Doenças Crônicas foi de 100,70 óbitos por 100 mil habitantes. Nesse estudo variou entre 0 e $100 \%$ entre as áreas de abrangência das Unidades de Saúde, o que significa dizer que em duas Unidades de Saúde, todos os óbitos relacionados à hipertensão arterial sistêmica e ao diabetes ocorreram na população entre 20 e 59 anos. O estudo de Drumond Jr. ${ }^{19}$ apresentou variação para esse coeficiente de 7,2 a $55,2 \%$ entre os distritos de saúde da cidade de São Paulo. Onze Unidades de Saúde apresentaram coeficientes maiores que a média do município no ano de 2004, coincidindo também com o mapa de áreas de maior vulnerabilidade social do IPVS.

O Coeficiente de Mortalidade por Causas Externas foi de 51,59 óbitos por 100 mil habitantes. Nesse estudo apresentou resultados entre 0 e 109,7 óbitos por 100 mil habitantes.

Os achados do estudo de Drumond Jr. ${ }^{19}$ no município de São Paulo, no ano de 2004, utilizando a mesma metodologia, variou para as Distritais de Saúde da capital de 32,7 a 101,8 óbitos por causas externas por 100 mil habitantes. Treze das 24 Unidades apresentaram Coeficiente de Mortalidade por Causas Externas superiores à média do município de Araraquara, sendo que 11 delas correspondem às regiões da periferia que, segundo o IPVS, representam áreas de maior vulnerabilidade.

O município de Araraquara apresentou taxa de alfabetização segundo o IBGE de $94,8 \%$ no ano de 2000 . Os resultados desse estudo apontaram variação nas Unidades de Saúde entre 68,2 e 97,8\%. Apesar do bom desempenho do município, vale ressaltar que, ao observar individualmente as áreas intraurbanas, foram identificados diferenciais importantes. As populações com baixa escolaridade em geral tendem a ter condições de vida mais desfavoráveis, pois enfrentam maiores dificuldades na obtenção de um posto de trabalho e no cuidado com a saúde, por exemplo. Logo, é de se esperar uma vulnerabilidade ascendente conforme diminua o grau de escolaridade dos responsáveis pelos domicílios ${ }^{21}$.

Para o componente RMRD encontraram-se importantes diferenças variando entre $\mathrm{R} \$ 302,89$ e $\mathrm{R} \$ 1.781,83$. A renda per capita do município, no ano de 2001 foi de $\mathrm{R} \$ 441,88^{21}$. Considerando esse componente, as desigualdades no território acompanharam também os piores desempenhos nos outros componentes desse estudo, apontando assim para a estreita relação entre os mesmos. A importância da renda em relação aos indicadores de saúde é apontada no Relatório Mundial de Saúde da $\mathrm{OMS}^{22}$, onde são descritas as tendências globais de sobrevivência das crianças e na expectativa de vida. $\mathrm{O}$ aumento nas desigualdades com relação à renda implica maior desigualdade na sobrevivência das crianças, sendo que o reflexo desse padrão de crescimento desigual sustenta as crescentes disparidades entre os mais e os menos saudáveis.

Para os componentes TARD e RMRD, também se observou que os piores resultados coincidem com a cartografia das regiões mais vulneráveis do IPVS. Quando consideradas apenas as variáveis estritamente relacionadas à saúde, parece não haver modificação no desempenho ao se comparar com o estudo do IPVS, que utiliza como componentes variáveis relacionadas à condição socioeconômica e ao ciclo de vida familiar. Seis das sete unidades com os piores resultados no estudo encontram-se nas áreas classificadas entre vulnerabilidade média e muito alta. Quando sobrepostas as representações cartográficas do IPVS e do ISM, observa-se que as áreas de abrangência cujos setores censitários apresentaram classificação acima de 3, (ou seja, 4, 5 e 6 - Vulnerabilidade Média, Alta e Muito Alta) no IPVS correspondem a áreas periféricas do município, onde sete das oito Unidades com as piores classificações no ISM coincidem com áreas de maior vulnerabilidade no IPVS. Os resultados finais obtidos pelas Unidades de Saúde no ISM variaram entre 0,38 (menor desempenho) e 0,81 (maior desempenho), demonstrando importantes 
diferenças na capacidade de atuação dessas Unidades no sentido de buscar a equidade da assistência, pois apresentam formas muito distintas na prestação da assistência particularmente no que se refere à qualidade, produzindo desigualdade, sobretudo para aqueles que mais necessitam ${ }^{7}$

O Quadro 3, abaixo, ilustra a comparação entre o valor obtido na classificação da área de abrangência no ISM e a classificação da mesma área no IPVS, em 2000.

Os resultados obtidos no presente estudo vão ao encontro das proposições de Akerman ${ }^{8}$, que destaca a importância dos problemas das nossas cidades não serem abordados de maneira isolada, para a produção de resultados capazes de dar respostas às necessidades das pessoas. As proposições de $\mathrm{Mendes}^{2}$ na mesma direção apontam para a necessidade do uso dos indicadores compostos que demonstrem os diferenciais intraurbanos, dando conta dos fenômenos de interação e sinergismo entre os componentes utilizados no estudo e reforçados pelo estudo do Índice Paulista de Vulnerabilidade Social.

Esses resultados reforçam Akerman ${ }^{8}$, segundo o qual a adoção de indicadores

Quadro 3 - Comparação entre o valor final obtido por cada área de abrangência das Unidades de Saúde no Índice de Saúde Modificado e a classificação dos setores censitários das respectivas áreas de abrangência no Índice Paulista de Vulnerabilidade Social - IPVS, 2000.

Chart 3 - Comparison between the final value obtained by each Health Unit in the Health Index and the Modified classification of census tracts within their areas of coverage in the Index of Social Vulnerability - IPVS, 2000.

\begin{tabular}{|lccc|}
\hline $\begin{array}{l}\text { Áreas de abrangência } \\
\text { das unidades de } \\
\text { saúde }\end{array}$ & $\begin{array}{c}\text { Índice de saúde } \\
\text { modificado }\end{array}$ & $\begin{array}{c}\text { Classificação final } \\
\text { SESA }\end{array}$ & $\begin{array}{c}\text { Classificação dos setores } \\
\text { censitários da área de } \\
\text { abrangência no IPVS }\end{array}$ \\
CMS PAULISTANO & 0,81 & $1^{\circ}$ & 1,2 e 3 \\
PSF B. ANDRADA*** & 0,80 & $2^{\circ}$ & 1 e 2 \\
PSF BELA VISTA*** & 0,80 & $2^{\circ}$ & - \\
PSF MARIVAN & 0,73 & $3^{\circ}$ & - \\
CMS S. ANGELINA & 0,72 & $4^{\circ}$ & 3 \\
CMS SANTA LÚCIA & 0,72 & $5^{\circ}$ & 2 e 3 \\
PSF VALE DO SOL & 0,67 & $5^{\circ}$ & 1,2 e 3 \\
CMS SELMI DEIIV & 0,67 & $6^{\circ}$ & 2 e 3 \\
CMS CECAP & 0,66 & $6^{\circ}$ & 1,2 e 5 \\
PSF HORTENSIAS & 0,64 & $7^{\circ}$ & 2,3 e 5 \\
CMS JD. AMERICA & 0,62 & $8^{\circ}$ & 5 e 6 \\
CMS JD. IGUATEMI & 0,60 & $10^{\circ}$ & 2,3 e 5 \\
CMS V. MELHADO & 0,60 & $10^{\circ}$ & 3 e 4 \\
CMS LARANJEIRAS & 0,58 & $11^{\circ}$ & 1,2 e 3 \\
CMS VILA XAVIER & 0,57 & $12^{\circ}$ & $1,2,4$ e 5 \\
PSF JD. PINHEIROS & 0,51 & $13^{\circ}$ & 2 e 3 \\
PSF MARIA LUIZA & 0,47 & $14^{\circ}$ & 3,4 e 5 \\
CMS Y. ÓPICE & 0,46 & $15^{\circ}$ & 2,3 e 4 \\
CMS SELMI DEII & 0,43 & $16^{\circ}$ & $2,3,4$ e 5 \\
PSF A. DO PAIOL & 0,41 & $17^{\circ}$ & $2,3,4,5$ e 6 \\
PSF V. BIAGIONI & 0,40 & $18^{\circ}$ & 2,4 e 6 \\
PSF IEDA & 0,39 & $19^{\circ}$ & 3 \\
CMS PQ.S. PAULO & 0,38 & $20^{\circ}$ & 4,5 e 6 \\
\hline
\end{tabular}

***Nota: A presente classificação refere-se somente aos setores urbanos, as áreas assinaladas são áreas rurais as quais não são contempladas no IPVS.

***Note: The present ranking refers to urban sectors exclusively and the areas marked are rural, thus not being included in the IPVS. 
compostos pode ser uma estratégia para mostrar as diferenças nos espaços urbanos, buscando necessidades, possibilidades de soluções e caminhos para se equacionarem essas diferenças. Tobar et al. ${ }^{23}$ consideram três dimensões básicas no sentido de diminuir as iniquidades sociais: acesso a serviços de saúde, equidade na dotação de recursos financeiros e equidade nos resultados, ou seja, independentemente das condições socioeconômicas, as classes sociais devem alcançar resultados equânimes. Equidade aqui é entendida como a superação das diferenças evitáveis e derivadas de injustiças sociais.

Esses resultados que apontam importantes diferenciais entre as áreas de abrangência das Unidades de Saúde, sobretudo nas áreas periféricas, abrem assim possibilidades para o município de Araraquara/ SP identificar e implementar soluções no sentido de minimizar e equacionar essas diferenças , trilhando o caminho da equidade.

\section{Considerações Finais}

O presente estudo voltou-se para a análise das condições de saúde de um município do interior do Estado de São Paulo, valendo-se de indicadores compostos elegendo variáveis epidemiológicas de relevância para a saúde coletiva, capazes de revelar aspectos sócio-sanitários presentes no processo saúde-doença-cuidado.

Para cada um dos componentes adotados revelaram-se padrões de desigualdade na saúde onde os piores resultados coincidiram com as regiões periféricas do município correspondentes às áreas de maior vulnerabilidade social apontadas no IPVS.

O ISM, sendo um indicador composto, permitiu tanto a análise agrupada dos seus componentes quanto a apreciação individualizada de cada um deles e por área de abrangência da unidade de saúde, aproximando-se mais da realidade local. Esse enfoque pode permitir que se avalie com maior precisão os riscos inerentes à saúde e a pobreza aos quais estão submetidos os cidadãos, o que foi possível através do uso de indicadores de saúde que constituem potentes ferramentas no processo de avaliação e monitoramento na identificação de desigualdades territoriais na saúde.

Ao evidenciar o impacto das desigualdades loco-regionais na prestação da assistência e nas condições de vida a que os cidadãos estão submetidos, particularmente na Atenção Básica em seu sentido mais amplo, reforça a compreensão de que as diferentes necessidades dos distintos grupos sociais têm implicações no adoecer e morrer desses grupos e indivíduos.

Acredita-se que o esforço de sistematizar e produzir conhecimentos sobre essa realidade possa se constituir em uma contribuição imediata ao município de Araraquara, envolvendo não só a Secretaria Municipal de Saúde em seus vários níveis gestão, mas também, profissionais e comunidade ativa - que no seu dia a dia leva à frente o desafio de uma assistência ainda pouco equânime.

O uso dessa metodologia pode, ainda, servir para realidades de outros municípios ou serviços, que poderão se valer de indicadores locorregionais, por meio de componentes que traduzam a sua realidade, experiência ou necessidade. Poderão, assim, dispor de mais subsídios para refletir a respeito dos elementos que induzem diferentes desempenhos da assistência à saúde, bem como implantar essa proposta como instrumento de avaliação e monitoramento permanentes nas áreas de abrangência dos seus serviços.

O estudo apresenta algumas limitações, tais como: estar condicionado à qualidade e precisão da base de dados disponível; ter tomado como referência um curto período de tempo (um ano); não permitir comparações entre os resultados obtidos em municípios de realidades diferentes. Algumas dessas limitações podem ser superadas mediante a realização de estudos novos e mais específicos. Outras, mediante a formação e incorporação de uma nova cultura avaliativa.

Como recomendação imediata à gestão municipal, evidencia-se a importância de maior articulação das áreas sociais e ações intersetoriais, a ampliação da Estratégia 
Saúde da Família, investimentos para a melhoria do sistema de informações, com a adoção de recursos tecnológicos que permitam trabalhar com as informações no menor nível de desagregação possível de dados, de forma a melhorar a compreensão da realidade local, buscando o fortalecimento do controle social sobre essa realidade.
Outra possibilidade que se abre por meio de estudo dessa natureza é a de servir como referência para acompanhamento periódico dos resultados de desempenho das diferentes Unidades de Saúde, por meio da sua mobilidade na classificação dos serviços, conforme forem introduzidas as mudanças envolvidas nos seus diferentes componentes.

\section{Referências}

1. Carvalho G, Rosemburg CP, Buralli KO. Avaliação de Ações e Serviços de Saúde. Revista O Mundo da Saúde 2000; 24(1): 72-5.

2. Mendes EV. Organização da Saúde no Nível Local. $1^{\mathrm{a}}$ ed. São Paulo: Hucitec; 1998.

3. São Paulo. Secretária Estadual da Saúde. Coordenadoria de Planejamento em Saúde. Manual de Orientações para os Indicadores de Gestão. SP: SES: CPS; 2002.

4. Akerman M, Stephens C, Campanario P, Maia PB. Saúde e meio ambiente: uma análise de diferenciais intraurbanos enfocando o Município de São Paulo, Brasil. Rev Saúde Pública 1994; 28(4): 320-5.

5. Berlinguer G. Ética da saúde. 2a ed. São Paulo: Hucitec; 1996.

6. Sposatti A. Mapa da exclusão/inclusão social da cidade de Santo André. Santo André: Pontifícia Universidade Católica de São Paulo; 2000.

7. Organização Mundial da Saúde, The World Health Report 2008: Primary Health Care Now More Than Ever. Organização Mundial da Saúde; 2008. 156p.

8. Akerman M. A construção de indicadores compostos para projetos de cidade saudáveis: um convite ao pacto transetorial. In: Mendes EV (org.). Organização da Saúde no Nível Local. $1^{\text {a }}$ ed. São Paulo: Hucitec; 1998. cap.1, p. 319-35.

9. Chiesa AM, Westphal MF, Kashiwagi NM.

Geoprocessamento e a promoção da saúde. Rev Saúde Pública 2002; 36(5): 559-67.

10. Fundação Seade. IPVS. Espaços e dimensões da pobreza nos municípios do Estado de São Paulo. Região Administrativa Central; 2000.

11. Drumond M, Jr. Índice de Saúde. Coordenação de Epidemiologia e Informação, Secretaria Municipal de Saúde do município de São Paulo; 2002.

12. Kaufman RA. Planificación de Systemas Educativos. México: Tnllas; 1973.

13. Samohyl, R. Ferramentas Estatísticas de Qualidade. In: Carvalho, MM. Gestão da Qualidade: teoria e casos. Rio de Janeiro: Elsevier; 2005.
14. FAPESP. Projeto Dinâmica Social, Qualidade Ambiental e Espaços Intraurbanos em São Paulo. FAPESP; 2000.

15. Almeida MF. Descentralização de Sistemas de Informação e o Uso das Informações a Nível Municipal. IESUS 1998; VII(3): 29.

16. Moreira ML. Sistema de Informação de Saúde: A Epidemiologia e a Gestão de Serviço. Saúde e Sociedade 1995; 4(1/2): 43-45.

17. Mattos ATR, Caccia-Bava MC. Repercussões da Implantação do Programa Saúde da Família no Município de Araraquara: Impactos e Perspectivas. Rev Bras Med Fam Com 2004; 1(1): 36-45.

18. São Paulo. Secretaria de Estado da Saúde. Centro de Vigilância Epidemiológica Alexandre Vranjac. Tuberculose em números; 2004.

19. Drumond M, Jr. Painel de monitoramento das condições de vida e saúde e da situação dos serviços de saúde. Coordenação de Epidemiologia e Informação, Secretaria Municipal de Saúde do município de São Paulo; 2004.

20. Cruz MG. Estudo comparativo de alguns indicadores em municípios do Estado de São Paulo segundo a implantação da Saúde da Família. Rev Esc Enferm USP 2005; 39(1): 28-35.

21. São Paulo. Secretaria de Estado de Economia e Planejamento. IDH - Indicadores de desenvolvimento/2001 por municípios; 2001.

22. Organização Mundial da Saúde. The World Health Report 2008: Primary Health Care Now More Than Ever. Organização Mundial da Saúde; 2008. 156p.

23. Tobar F, Montiel L, Gaya R, Martínez E. Modelos Equitativos de Distribución de Recursos Sanitarios. In: Buitrago, RIR. La política de financiación de los sistemas de salud y la equidad de género en el estado de salud. Tesis (Magíster en administración de salud). Pontificia Universidad Javeriana: Bogotá; 2005. 213p.

Recebido em: 24/01/11 Versão final apresentada em: 13/01/12 Aprovado em: 24/04/12 